\title{
Investigating organic multilayers by spectroscopic ellipsometry: specific and non-specific interactions of polyhistidine with NTA self-assembled monolayers
}

\author{
Ilaria Solano ${ }^{1}$, Pietro Parisse ${ }^{2}$, Ornella Cavalleri ${ }^{*}{ }^{1}$, Federico Gramazio ${ }^{1}$, \\ Loredana Casalis ${ }^{2}$ and Maurizio Canepa ${ }^{1}$
}

\author{
Full Research Paper \\ Address: \\ ${ }^{1}$ Dipartimento di Fisica, Università di Genova, Via Dodecaneso 33, \\ Genova, Italy and ${ }^{2}$ Elettra Sincrotrone Trieste S.C.p.A., s.s. 14 km \\ 163,5 in Area Science Park, Basovizza, Trieste, Italy \\ Email: \\ Ornella Cavalleri* - cavalleri@fisica.unige.it \\ * Corresponding author \\ Keywords: \\ His-tag; nitrilotriacetic acid (NTA); protein binding; self-assembled \\ monolayers (SAMs); spectroscopic ellipsometry
}

\author{
Beilstein J. Nanotechnol. 2016, 7, 544-553. \\ doi:10.3762/bjnano.7.48 \\ Received: 02 December 2015 \\ Accepted: 31 March 2016 \\ Published: 13 April 2016 \\ This article is part of the Thematic Series "Organized films". \\ Associate Editor: J. Lahann
}

(c) 2016 Solano et al; licensee Beilstein-Institut. License and terms: see end of document.

\begin{abstract}
Background: A versatile strategy for protein-surface coupling in biochips exploits the affinity for polyhistidine of the nitrilotriacetic acid (NTA) group loaded with Ni(II). Methods based on optical reflectivity measurements such as spectroscopic ellipsometry (SE) allow for label-free, non-invasive monitoring of molecule adsorption/desorption at surfaces.

Results: This paper describes a SE study about the interaction of hexahistidine (His 6 ) on gold substrates functionalized with a thiolate self-assembled monolayer bearing the NTA end group. By systematically applying the difference spectra method, which emphasizes the small changes of the ellipsometry spectral response upon the nanoscale thickening/thinning of the molecular film, we characterized different steps of the process such as the NTA-functionalization of Au, the adsorption of the His 6 layer and its eventual displacement after reaction with competitive ligands. The films were investigated in liquid, and ex situ in ambient air. The SE investigation has been complemented by AFM measurements based on nanolithography methods (nanografting mode).

Conclusion: Our approach to the SE data, exploiting the full spectroscopic potential of the method and basic optical models, was able to provide a picture of the variation of the film thickness along the process. The combination of $\delta \Delta_{i+1, i}(\lambda), \delta \Psi_{i+1, i}(\lambda)($ layer-addition mode) and $\delta \Delta_{i, i+1}^{\dagger}(\lambda), \delta \Psi^{\dagger} i_{i, i+1}(\lambda)$ (layer-removal mode) difference spectra allowed us to clearly disentangle the adsorption of $\mathrm{His}_{6}$ on the Ni-free NTA layer, due to non specific interactions, from the formation of a neatly thicker His ${ }_{6}$ film induced by the Ni(II)-loading of the NTA SAM.
\end{abstract}




\section{Introduction}

The design of biosensing devices requires precise immobilization, with controlled orientation, of functional proteins on a surface, generally consisting of a substrate functionalized with a molecular receptor layer [1,2].

After seminal works [3], the interaction of chelated Ni ions with oligohistidine peptides has been largely implemented over the years for the affinity purification of recombinant proteins [4-6], and for the development of bioanalytical surfaces [7-12]. To this purpose, self-assembled monolayers (SAMs) terminated with the nitriloacetic acid (NTA) group, after loading with nickel ions (Ni(II)), provide platforms able to specifically bind his-tag proteins [13-18] and enzymes that retain their activity upon immobilization [19-21]. The affinity between the adsorbent surface and the protein can be modulated, e.g., by using multi-chelator constructs $[22,23]$ or double hexahistidine tags [24].

Among applications, the NTA-Ni(II)-His coupling has been exploited for the functionalization of nanoparticles [25-27] and atomic force microscopy (AFM) tips [28] as well as for more biologically oriented applications such as the delivery of polymeric gene vehicles [29] or for the design of NTA-functionalized fluorescent probes able to track cellular events in situ by targeting specific His-tagged proteins [30,31].

Beyond selectivity, the reversibility of the NTA-Ni(II)-His coupling upon reaction with competitive ligands [32] makes this strategy a versatile system for the development of highly sensitive, specific and renewable sensing devices [33].

A high-performance sensor needs to integrate a specific binding scheme with a sensitive, non-destructive transduction of the binding event into a measurable signal. Optical methods based on optical reflectivity, such as surface plasmon resonance (SPR) and ellipsometry, allow for label-free, non-invasive, in situ monitoring of molecular adsorption/desorption at surfaces [3440]. While SPR-based methods require supports with specific plasmonic properties, ellipsometry methods can be employed on a variety of reflecting solid surfaces. Spectroscopic ellipsometry (SE) can detect molecular adsorption on the receptor layer through the observation of thickness variations and, more specifically, through the spectroscopic characterization of UV-vis absorptions related to the analyte or to the receptor-analyte bond.

At variance with more traditional fields of application of SE [41-43], a reliable analysis of SE data on ultrathin films, at the nanometer scale thickness, must rely on the evaluation of the fine changes of the ellipsometry response upon the thickening/ thinning of the molecular film. Such variations can be emphasized looking at so-called difference spectra [44], $\delta \Psi_{i+1, i}(\lambda)$, $\delta \Delta_{i+1, i}(\lambda)$, where the suffix labels the difference between the spectra taken after and before the addition of the $(i+1)$-th layer in a multilayer stack.

Our approach, developed in recent years $[45,46]$, aims to exploit, under an unconventional perspective, the full spectroscopic potential of difference spectra. Regarding the important class of thiolate SAMs on flat gold films, careful analysis of $\delta$-spectra allowed us to disentangle spectral features related to the SAM and to the S-Au interface region [46] as well as to identify fingerprints of molecular UV-vis absorptions [47,48], with sub-monolayer sensitivity.

In a very recent work we successfully combined our approach with thickness determination based on AFM measurements exploiting smart nanolithography methods, such as the so-called nanoshaving [49], that considerably helped to reduce the uncertainty related to the correlation between thickness and refractive index that is typical of ultrathin films.

A step forward, we intend to apply the same methods to control the adsorption of the analyte layer (AL) on the receptor layer (RL), eventually distinguishing between specific and non-specific binding. In this article we describe a study about the interaction of $\mathrm{His}_{6}$ on gold substrates functionalized with NTA (Figure 1). While on simple NTA-terminated films only nonspecific adsorption of $\mathrm{His}_{6}$ is expected, the loading with $\mathrm{Ni}$ (II) endows the RL with specific affinity towards $\mathrm{His}_{6}$. We investigated the sequence of steps of the process (schematically sketched in Figure 1) beginning from the functionalization of $\mathrm{Au}$ until the final regeneration of the NTA layer through displacement of His $_{6}$. To this aim we will introduce convenient $\delta \Delta_{i^{\prime}, i+1}^{\dagger}(\lambda)$ and $\delta \Psi_{i^{\prime}, i+1}^{\dagger}(\lambda)$ difference spectra where the suffix labels the difference between the spectra taken after and before the removal of the $(i+1)$-th layer in a multilayer stack.

The systems were investigated in situ in liquid, and ex situ in ambient air. The experiments aimed to explore the variation of film thickness after convenient stages of the process as well as to detect spectral features that could be eventually related to the specific NTA-Ni(II)-His ${ }_{6}$ interaction. The SE investigation has been usefully complemented by AFM measurements based on SAM nanolitography (both shaving and grafting modes).

\section{Results and Discussion \\ Substrate functionalization: the NTA-SAM}

The first step of the experiment consisted of the formation and the characterization of the NTA-terminated precursor SAMs. 
(a)

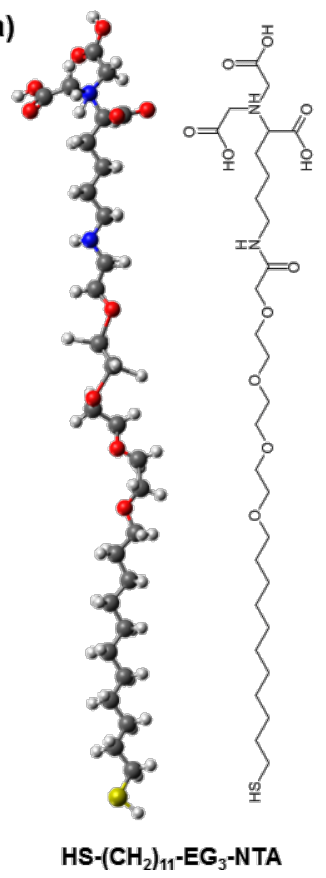

(b)

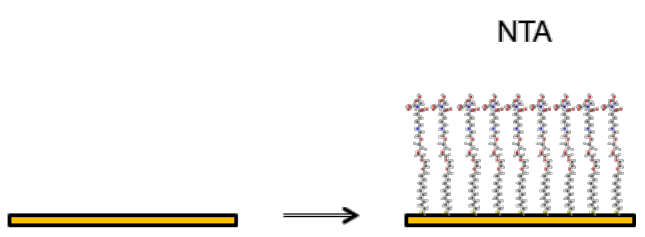

(1)

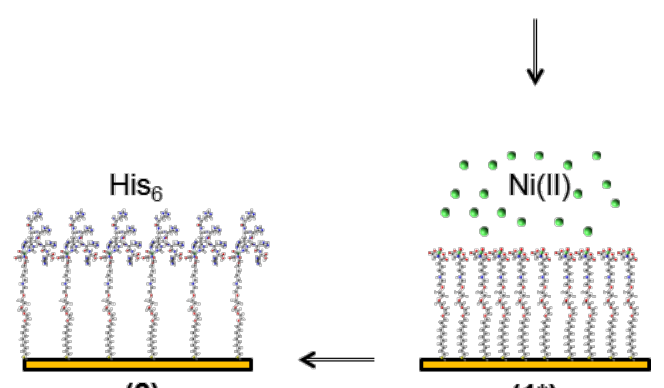

(2)

$\left(1^{*}\right)$

Figure 1: (a) Chemical structure of the HS-C11-EG3-NTA (NTA1) molecule; (b) sketch of the steps of sample preparation: (0) substrate preparation, (1) formation of the NTA SAM, $\left(1^{*}\right) \mathrm{Ni}(\mathrm{II})$ loading and (2) adsorption of $\mathrm{His}_{6}$. Step (1 $\left.{ }^{*}\right)$ was skipped for a subset of samples in order to check non-specific $\mathrm{His}_{6}-\mathrm{NTA}$ interactions. The ellipsometry analysis focused on steps (0), (1), (2).

Details on the preparation are reported in the Experimental section. Note that an accurate characterization of the precursor receptor SAM is a necessary prerequisite for a reliable interpretation of measurements about adsorption of analytes. Ex situ SE $\delta \Delta_{1,0}$ and $\delta \Psi_{1,0}$ spectra are presented in Figure 2 for two types of SAMs (HS-C11-EG3-NTA, NTA1, and HS-C16-EG3-NTA, NTA2). The suffixes 1 and 0 of $\delta \Delta_{1,0}$ and $\delta \Psi_{1,0}$ correspond to the SAM-Au and to the Au system, respectively. The results from NTA SAMs are compared with those obtained recently for oligo(ethylene glycol)-terminated alkanethiols, HS-C11-EG3 (T-OEG3) and HS-C11-EG6 (T-OEG6), for which the thicknesses were accurately determined by SE and AFM nanolithography [49].

The shape of the NTA spectra closely resembles that of T-OEG SAMS and does not show any evident features typical of intrinsic molecular optical absorptions [47,50]. Thus, the data are presented in comparison with a set of simulations based on the model of a transparent film (TF) onto the substrate $(\mathrm{Au})$, assuming for TF a Cauchy-type dispersion formula [42]:

$$
n=n_{\mathrm{TF}}+\frac{B\left[\mu \mathrm{m}^{2}\right]}{\lambda^{2}} ; \quad \kappa=0
$$

The shaded areas in Figure 2 span the representative range of $1.35 \leq n_{\mathrm{TF}} \leq 1.50$ [51], for three indicative values of the film thickness $d_{\mathrm{TF}}$. The coefficient $B$ was set to the value found in the analysis of T-OEG molecules (0.005) [49]. Note that the extension of the shaded bands provide an intuitive picture of the well-known correlation between refractive index and thickness that is typical for ultrathin films.

Systematic studies carried out in our group and, in particular, the analysis of the TF|Au model performed in [49] showed that $\delta \Delta_{1,0}$ and $\delta \Psi_{1,0}$ spectra provide a somewhat complementary information. While the $\delta \Delta_{1,0}$ spectra are sensitive to the properties of film + interface throughout the whole spectral range, the $\delta \Psi_{1,0}$ spectra show an interesting behavior in connection with the high reflectivity onset of $\mathrm{Au}$ which is evident in $\Psi$ spectra (Figure 2b) between 500 and $550 \mathrm{~nm}$.

Above this threshold, going towards the NIR, the TF|Au model predicts that $\Psi$ tends to assume values close to $45^{\circ}$ insensitive to transparent films: Simulated $\delta \Psi_{1,0}$ values vanish. Negative experimental $\delta \Psi_{1,0}$ values are due only to the absorptive properties of the interface layer, in connection with the formation of a strong molecule-gold bond [46,52].

In contrast, below the threshold, $\delta \Psi_{1,0}$ spectra bear substantial information only about the transparent part of the film [49]. 
(a)
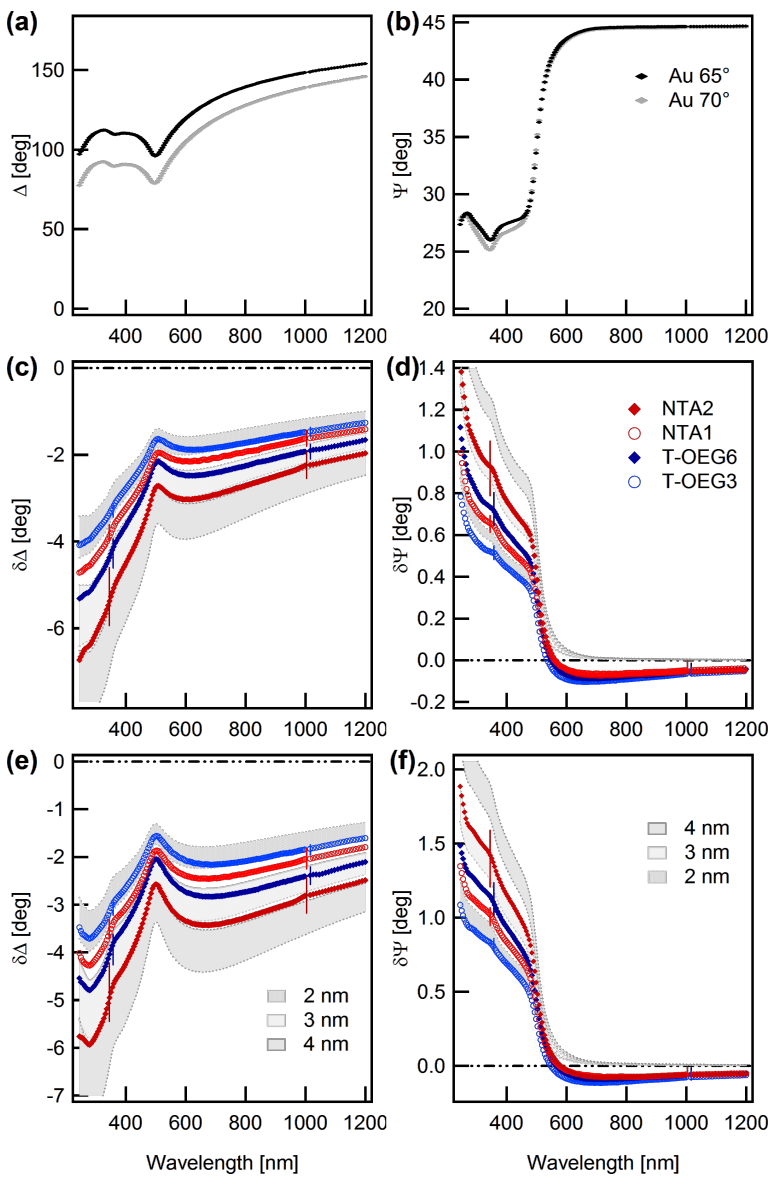

Figure 2: $(a, b)$ Ex situ experimental SE spectra obtained on gold substrates. (c-f) Ex situ experimental difference spectra $\left(\delta \Delta_{1,0}=\Delta_{1}-\Delta_{0}\right.$, $\delta \Psi_{1,0}=\Psi_{1}-\Psi_{0}$ ) obtained for NTA2 (red full diamonds) and NTA1 (red open circles) SAMs. Panels $\mathrm{c}$ and $\mathrm{d}: 65^{\circ}$ angle of incidence, panels e and $\mathrm{f}: 70^{\circ}$ angle of incidence. Data from T-OEG6 and T-OEG3 SAMs [49] are also shown for reference. Thin vertical bars represent the experimental uncertainty related to data dispersion over the investigated samples. Shaded areas illustrate the results of simulations based on the TF|Au model, for three values of the SAM thickness $\left(d_{\mathrm{TF}}=2,3\right.$ and $\left.4 \mathrm{~nm}\right)$. For $\delta \Delta_{1,0}$ patterns (left) the top and bottom borders of the shadings correspond to $n_{\mathrm{TF}}=1.35$ and 1.50 , respectively. For the $\delta \Psi_{1,0}$ spectra (right panels) the order is reversed.

Further, compared to the $\delta \Delta_{1,0}$ spectra, $\delta \Psi_{1,0}$ spectra are less affected by the correlation between $n_{\mathrm{TF}}$ and $d_{\mathrm{TF}}$.

Thus, from careful inspection of the $\delta \Psi_{1,0}$ patterns in Figure 2, and considering the uncertainties of experiment and model, one can estimate $d_{\mathrm{TF}}$ values of $2.2 \pm 0.3$ and $3.1 \pm 0.3 \mathrm{~nm}$ for the NTA1 and NTA2 SAMs, respectively. Negative $\delta \Psi_{1,0}$ values, which are substantially common to all the SAMs of Figure 2 and closely comparable to other well-structured thiolate- $\mathrm{Au}$ SAMs $[46,47,53,54]$, can be effectively reproduced by simulations considering an effective interface layer [55] with effective thickness $d_{\mathrm{I}}$ of the order of $0.2 \mathrm{~nm}[46,49]$. The sum $D$ of $d_{\mathrm{I}}$ and $d_{\mathrm{TF}}(2.4 \pm 0.3$ and $3.3 \pm 0.3 \mathrm{~nm}$ for the NTA1 and NTA2
SAMs, respectively) accounts for the total film thickness that is reflected in the $\delta \Delta_{1,0}$ spectra.

The AFM images of Figure $3 \mathrm{a}$ are representative of experiments of nanografting of T-OEG6 molecules in NTA SAMs. The latter were deposited with the same procedures applied in the SE measurements. The grafted areas are darker than the surrounding SAM carpet, indicating a lower height level. The histograms reported in Figure 3c show distributions that can be fitted by the superposition of two Gaussian curves. One, related to the T-OEG6 surface is taken as the reference; the second corresponds to the surface of the NTA SAM. The separation between the centers of the Gaussian curves provides $\Delta h_{\mathrm{NTA}-\mathrm{TOEG} 6}$, the height difference between the NTA and T-OEG6 SAMs.

After repeating this procedure for ten grafted patches we were able to obtain an average value of $\Delta h_{\text {NTA2-TOEG6 of }}$ $0.95 \pm 0.15 \mathrm{~nm}$ (Figure 3d). Regarding NTA1 SAMs, the contrast with the nanografted T-OEG6 patches is much lower (Figure $3 \mathrm{~b}$ ). In this case the analysis of images provides $\Delta h_{\text {NTA1-TOEG6 }}$ of $0.2 \pm 0.1 \mathrm{~nm}$ (Figure 3d). Combining these findings with the AFM results regarding the height of T-OEG6 SAMs presented in [49], which amounted to $2.4 \pm 0.3 \mathrm{~nm}$, we obtain for the NTA1 and NTA2 films an AFM thickness of $2.6 \pm 0.4$ and $3.4 \pm 0.5 \mathrm{~nm}$, respectively.

The height difference between NTA2 and NTA1 SAMs measured with AFM $\left(\Delta h_{\mathrm{NTA} 2-\mathrm{NTA} 1}=0.8 \mathrm{~nm}\right)$ and the thickness difference obtained by SE $\left(\Delta D_{\mathrm{NTA} 2-\mathrm{NTA} 1}=0.9 \mathrm{~nm}\right)$ agree with the nominal length difference between the two molecules, of about $0.8 \mathrm{~nm}$. However, although AFM and SE findings are compatible within the experimental uncertainty, the measurements by AFM yield height values that are systematically larger than the ellipsometry thickness. Small discrepancies between the two methods appear conceivable and even expected for several reasons. First of all, we need to consider that the NTA head is more charged than the OEG termination and can be responsible of an increased electrostatic repulsion and therefore of an increased height during the measurements in liquid. Moreover, we should consider systematic differences regarding the macroscopic area probed by the optical beam in SE vs the microscopic area probed by AFM and the different environment of the measurements (liquid for AFM, air for SE).

Note that SE does not provide direct information about molecular orientations in the SAM and about the SAM organization. Such properties should be probed through surface-sensitive infrared spectroscopy methods such as reflection-absorption infrared spectroscopy [56]. Nevertheless, the values of film thickness determined by SE and AFM testify the formation of 

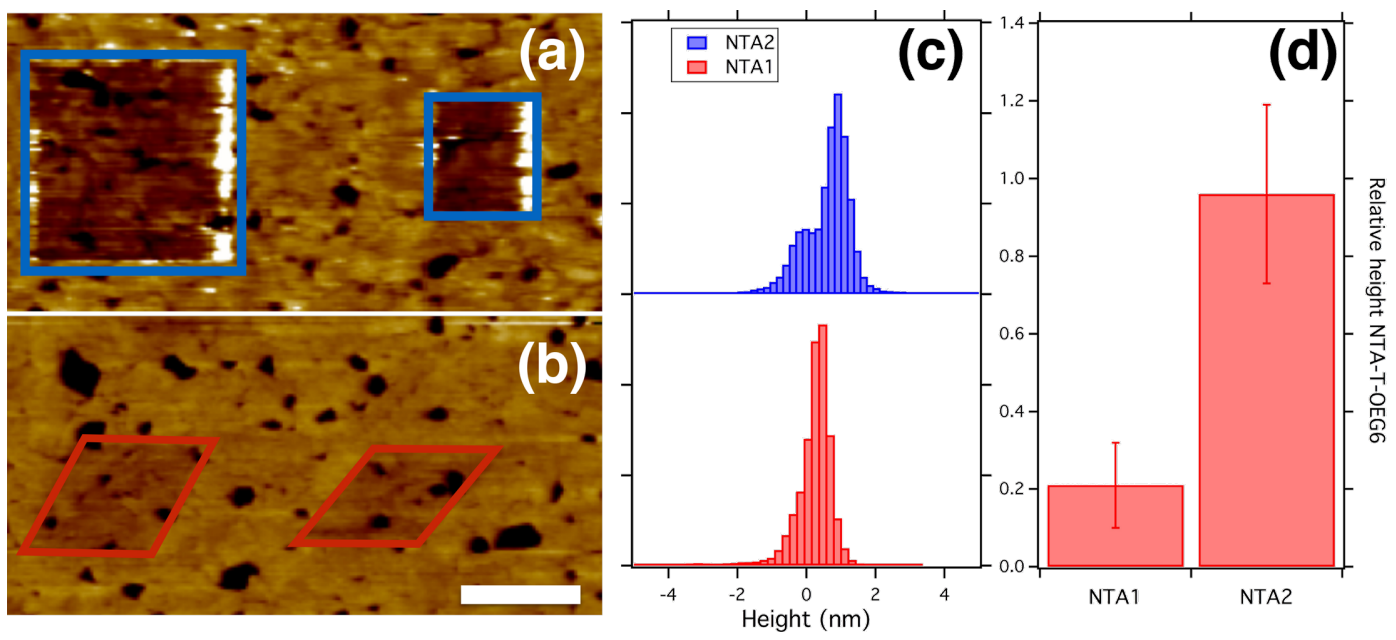

Figure 3: (a) Atomic force microscopy image showing two nanografted patches of T-OEG6 in a NTA2 SAM (b) the same for a NTA1 SAM. The white scale bar corresponds to $1 \mu \mathrm{m}$. (c) Height histograms corresponding to the AFM images in (a) and (b). The reference has been set at the center of the Gaussian distribution related to the squared dark areas in the images. (d) Synthesis of histogram analysis for the NTA1 and NTA2 samples investigated providing the average values of $\Delta h_{\mathrm{NTA}-\mathrm{TOEG} 6}$ and $\Delta h_{\mathrm{NTA} 1-\mathrm{TOEG} 6}$ (for details see the text).

compact NTA SAMs, which can be used for second-layer adsorption experiments.

\section{His $_{6}-$ NTA interaction}

Exploratory checks showed that the variations induced by $\mathrm{Ni}(\mathrm{II})$ loading on the NTA/Au SE spectra were very small. Thus, we decided to study the $\mathrm{His}_{6}$ adsorption (step (2) in Figure 1) by referencing the difference spectra to the data of the NTA film. Specific or non-specific interactions of $\mathrm{His}_{6}$ are therefore probed through a comparison of the results obtained with or without the $\mathrm{Ni}(\mathrm{II})$ pre-loading. We adopted preparation protocols described in literature [57], which are summarized in the Experimental section.

$\delta \Delta_{2,1}$ and $\delta \Psi_{2,1}$ difference spectra shown in Figure 4a,b are representative of the observed behavior. Despite the reduced refractive index mismatch between the film and the ambient and the use of cell windows tending to lower the signal-to-noise ratio, the emerging picture appears rather sharp.

In the case of Ni-free RL (light grey circles) we found very low $\delta \Psi_{2,1}$ and $\delta \Delta_{2,1}$ spectral values, over the whole wavelength range. These results suggest a very modest thickness increment. In facts, the formation of films uniformly spread on gold-like substrates, even if very thin, usually induces a well-defined relative maximum of $\delta \Delta_{i+1, i}$ at about $500 \mathrm{~nm}$ [46,52]. This is actually missing in the data, possibly indicating the formation of a low-coverage sub-monolayer [58], a situation that is compatible with non-specific $\mathrm{His}_{6}-\mathrm{NTA}$ interactions, in accordance with literature [10].

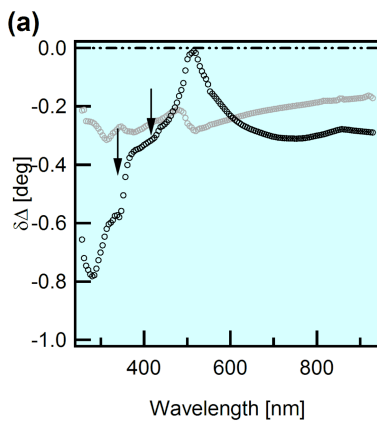

(b)

(c)

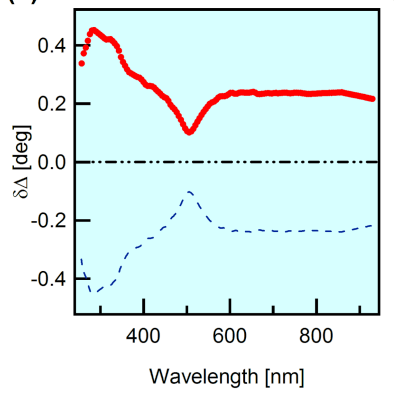

(d)

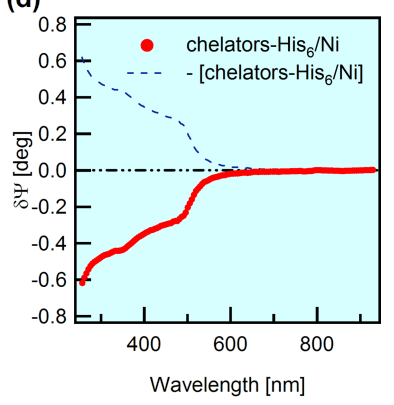

Figure 4: $\mathrm{His}_{6}$ adsorption on the NTA/Au system and subsequent treatment with imidazole/EDTA. The SE data are derived from in situ (buffer) measurements at $65^{\circ}$ angle of incidence. (a,b) $\delta \Delta_{2,1}$ and $\delta \Psi_{2,1}$ difference spectra; the suffixes 1 and 2 indicate the NTA1/Au system before and after the exposure to $\mathrm{His}_{6}$ molecules, respectively. Black and light grey symbols refer to measurements with and without $\mathrm{Ni}(\mathrm{II})$ loading, respectively. Arrows indicate possible weak optical absorptions (see the text for details). (c,d) Red symbols: $\delta \Delta_{1^{\prime}, 2}^{\dagger}, \delta \Psi_{1^{\prime},}^{\dagger}$ spectra; the suffixes 1 ' and 2 stand for the His ${ }_{6} / \mathrm{NTA} 1 / \mathrm{Au}$ system after and before the treatment with chelating agents. The blue dashed curves represent $-\delta \Delta_{1^{\prime}, 2}^{\dagger}$ and $-\delta \Psi_{1^{\prime}, 2}^{\dagger}$ spectra. 
Conversely, well-structured spectra were observed after the interaction of $\mathrm{His}_{6}$ with Ni-loaded NTA SAMs (black open circles), testifying the formation of a well-defined layer. Note in passing that $\mathrm{Ni}(\mathrm{II})$ loading and subsequent $\mathrm{His}_{6}$ adsorption have very little influence on the $\delta \Psi_{2,1}$ values above $550 \mathrm{~nm}$, which are overall positive and close to zero. This finding suggests that the percolation of Ni through the compact NTA SAM down to the $\mathrm{S}-\mathrm{Au}$ interface is likely negligible.

In order to add confidence about the formation of the $\mathrm{Ni}(\mathrm{II})-\mathrm{His}_{6}$ complex, we analyzed its interaction with competitive chelating agents eventually leading to the regeneration of the NTA film. Representative difference between spectra measured after and before the imidazole/EDTA treatment, for brevity $\delta \Delta_{1^{\prime}, 2}^{\dagger}$ and $\delta \Psi_{1^{\prime}, 2}^{\dagger}$, are shown in Figure 4c,d (red open circles).

The graphic choice in panels c and d emphasizes that $\delta \Delta_{1^{\prime}, 2}^{\dagger}$ and $\delta \Psi_{1^{\prime}, 2}^{\dagger}$ spectra are specular (with respect to the null level) to $\delta \Delta_{2,1}$ and $\delta \Psi_{2,1}$ spectra which have been considered until now (and in all our previous works on SAMs on Au). $\delta \Delta_{i+1, i}$ and $\delta \Psi_{i+1, i}$, were always associated with the addition of a molecular layer (for example the NTA layer on the Au substrate or the His $_{6}$ layer on the NTA/Au system). In the case of Figure 4c,d, positive $\delta \Delta_{1^{\prime}, 2}^{\dagger}$ and low-wavelength, negative $\delta \Psi_{1^{\prime}, 2}^{\dagger}$ (red curves) indicate a neat decrease of the thickness, to be associated with the removal of molecular layer $2\left(\mathrm{His}_{6}\right)$.

Further insight into thickening or thinning of the films following specific adsorption and removal of $\mathrm{His}_{6}$ was obtained from ex situ measurements. In Figure 5, $\delta \Delta_{2,1}$ and $\delta \Psi_{2,1}$ spectra (black symbols), related to the $\mathrm{His}_{6}$ adsorption, are compared with $\delta \Delta_{1^{\prime}, 1}$ and $\delta \Psi_{1^{\prime}, 1}$ spectra (grey symbols) obtained after the completion of the imidazole/EDTA treatment. Note that these difference spectra are referenced to the NTA/Au film. In the figure, we also report the $\delta \Delta_{1,0}$ and $\delta \Psi_{1,0}$ spectra from Figure 2, referenced to the bare gold substrate. Ideally, $\delta \Delta_{1^{\prime}, 1}$ and $\delta \Psi_{1^{\prime}, 1}$ should present values close to the zero. Indeed, they appear significantly lower than the $\delta \Delta_{2,1}$ and $\delta \Psi_{2,1}$ spectra, indicating the thinning of the film.

The ex situ measurements are also useful for an estimate of the second layer thickness exploiting its rough proportionality with the NIR $\delta \Delta_{2,1}$ values, considering the NTA/Au film as reference and performing the same type of analysis as that in Figure 2. With a reasonable guess for the $\mathrm{His}_{6}$ layer refraction index in the range of $1.35-1.40$, the layer thickness is estimated in the range of $1.5-1.7 \mathrm{~nm}$ range. Analogously, we can also estimate that the imidazole/EDTA treatment removed $\approx 75 \%$ of

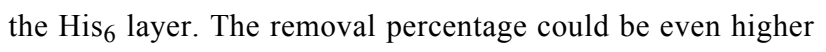
considering the conceivable effect of imidazole/EDTA resid-
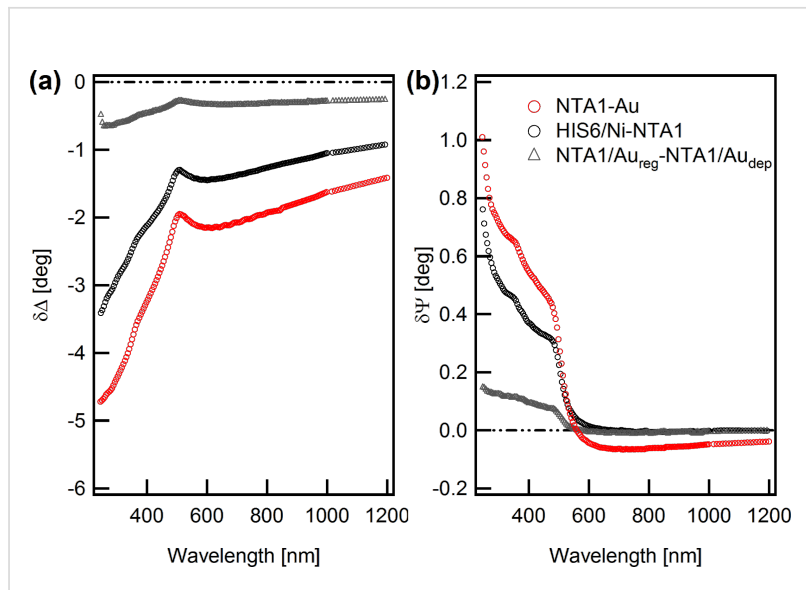

Figure 5: $\delta \Delta_{2,1}$ and $\delta \Psi_{2,1}$ spectra (black circles), related to the $\mathrm{His}_{6}$ adsorption, are compared with $\delta \Delta_{1 ', 1}$ and $\delta \Psi_{1 ', 1}$ spectra (grey triangles) obtained after the completion of the imidazole/EDTA treatment. These difference spectra are referenced to the NTA/Au system. $\delta \Delta_{1,0}$ and $\delta \Psi_{1,0}$ spectra associated to the deposition of NTA1 on the Au substrate (red circles) are also reported for comparison.

uals in $\delta \Delta_{1^{\prime}, 1}$ and $\delta \Psi_{1^{\prime}, 1}$ spectra. Therefore, the SE analysis of both in situ and ex situ data is able to follow the thickness variations induced by the addition/removal of the Ni(II)-His 6 layer.

An important step further would be the detection of spectral fingerprints of the $\mathrm{Ni}(\mathrm{II})-\mathrm{His}_{6}$ bond. In this respect, a comprehensive transmission spectroscopy study in solution reported optical absorptions of the $\mathrm{Ni}(\mathrm{II})-\mathrm{His}_{6}$ complex in the spectral range of 300-700 nm. Shape and intensity of absorption bands were found to depend on the $\mathrm{Ni}(\mathrm{II}) / \mathrm{His}_{6}$ concentration ratio and the $\mathrm{pH}$ value of the solution [15]. Indeed, in previous SE works on other SAMs on gold, we could detect optical molecular fingerprints as characteristic narrow-band dips in difference spectra. Specific examples are the intense Soret band observed in in situ spectra of cytochrome C SAMs $[48,50]$ or the UV absorptions of carbazole groups [47]. Detection of molecular fingerprints becomes definitely easier as the thickness scales up, as in the case of a $30 \mathrm{~nm}$ thick photochromic polymer film [59].

Due to index matching issues, observation of optical absorptions in SAMs becomes easier in liquid with refractive index close to that of the film $[48,50]$. A close look at in situ data (Figure 4) shows fine spectral features (indicated by arrows) which cannot be reproduced by a simple Cauchy model, and could be tentatively attributed to optical absorptions. However, these features are not intense and sharp enough for an unambiguous assignment, which would require, e.g., a check of $\mathrm{K}-\mathrm{K}$ consistency, as it was possible in previous experiments on other molecular systems $[48,50,59]$. Therefore the direct spectroscop-

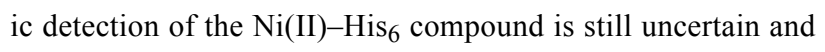
will require further investigation. 


\section{Conclusion}

We have applied spectroscopic ellipsometry to investigate the interaction of $\mathrm{His}_{6}$ with gold surfaces functionalized with a thiolate, OEG SAM bearing the NTA end-group, a system which is of recognized relevance for the design of a whole class of bio-sensing devices.

Indeed, ellipsometry was already employed to study related systems. In [37], about mixed layers of bis-NTA thiols with inert OEG thiols, the authors thoroughly reported on imaging ellipsometry measurements at a single wavelength. They exploited variations of $\Delta$ and $\Psi$ to obtain information about the thickness and homogeneity of the mixed layers while their functionalities were probed using imaging SPR. In [51] the NTA-Ni(II)-mediated immobilization of His $_{6}$-tagged green fluorescent protein on a silica surface was reported. Ellipsometry, at two wavelengths, was exploited mainly to characterize the precursor NTA SAM (about $15 \mathrm{~nm}$ thick) while the interactions with proteins of the NTA surface were studied by fluorescence methods. Spectroscopic ellipsometry was also frequently used on other NTA-related systems as an ancillary characterization method $[10,60,61]$.

To our knowledge this is the first time that SE has been used to comprehensively address the process, from the characterization of the precursor NTA layer to the study of the Ni(II)-mediated $\mathrm{His}_{6}$ absorption and the final regeneration of the NTA layer obtained by exposure to competitive chelating agents.

Our approach, progressively developed in recent years, is based on the careful analysis of SE difference spectra bearing information on the small changes of the ellipsometry response upon the thickening or thinning of molecular films with nanoscale thickness, and aims to fully exploit the rich broadband potential of SE, a point which marks the difference with single wavelength techniques, including SPR. Difference spectra, measured for hundreds of wavelengths ranging from the short UV to the NIR, proved able to show the variation of film thickness related to a complex process consisting of addition and removal of several organic layers.

Our investigation addressed the characterization of the NTA SAM on gold, exploiting the powerful cross-check with AFM measurements of the SAM height based on smart nanolitography methods. The SE data, which have been interpreted with basic models, provided a reliable evaluation of the thickness and optical properties of compact NTA SAMs. The accurate knowledge of the properties of the functionalizing SAMs constituted the necessary base for the second step of the experiment which regarded the investigation of the NTA reaction with His $_{6}$. The experiments were not conclusive regarding the direct spectroscopic detection of the NTA-Ni(II)- $\mathrm{His}_{6}$ bond, which will deserve further investigation. Nonetheless, the combination of $\delta \Delta, \Psi_{i+1, i}(\lambda)$ (layer-addition mode) and $\delta \Delta, \Psi_{i^{\prime}, i+1}^{\dagger}(\lambda)$ (layer-removal mode) allowed us to clearly disentangle the formation of a His ${ }_{6}$ film induced by Ni(II)-loading of the NTA SAM from the non-specific adsorption of $\mathrm{His}_{6}$ on the Ni-free NTA layer.

This investigation is intended to form a solid analytical background towards the study of layered systems of greater complexity such as the interaction of NTA with $\mathrm{His}_{6}$-tag proteins, or the DNA/DNA hybridization, which is ongoing in our laboratories. Further, this paper describes a quite general method, which can be applied to other forms of organized organic nano-multi layers on solid as well liquid reflecting substrates.

\section{Experimental \\ Gold substrates and reagents}

Gold films for optical measurements were purchased from Arrandee $^{\mathrm{TM}}$ (200 nm thick, deposited on glass with a $\mathrm{Cr}$ primer). For AFM measurements Ulman-type [62] ultra-flat stripped gold surfaces were preferred. Ultra-flat gold samples were prepared by UHV deposition of gold onto a freshly cleaned $\mathrm{Si}(111)$ wafer. A silicon sample was then glued on the gold surface and cured overnight at $130{ }^{\circ} \mathrm{C}$. Ultraflat and clean gold surfaces are obtained by mechanically stripping the silicon wafer substrate from the silicon gold sandwich $[49,62]$.

(1-Mercaptoundec-11-yl)tri(ethylene glycol) and (1-mercaptoundec-16-yl)tri(ethylene glycol) with NTA (for brevity NTA1 and NTA2) were purchased from Prochimia Surfaces. Hexahistidine $\mathrm{His}_{6}(840.85 \mathrm{~g} / \mathrm{mol})$ was purchased from GenScript. Ethylenediaminetetraacetic acid (EDTA), imidazole, tris(hydroxymethyl)aminomethane (Tris), $\mathrm{NaCl}$ and $\mathrm{NiCl}_{2}$ were purchased from Sigma-Aldrich.

\section{NTA SAM deposition and interaction with $\mathrm{His}_{6}$}

Clean Arrandee samples or freshly stripped Ulman gold samples were incubated for $24 \mathrm{~h}$ in $15 \mu \mathrm{M}$ NTA1 (NTA2) solution, using ethanol as solvent. For ex situ optical measurements the NTA/Au samples were rinsed with ethanol and dried with $\mathrm{N}_{2}$ flow. A subset of NTA/Au samples were transferred in a salt buffer solution (20 mM Tris and $150 \mathrm{mM} \mathrm{NaCl}, \mathrm{pH}$ 7.4) for subsequent characterization and treatments. After a first optical characterization in the new medium, the samples were immersed in 0.5 M EDTA ( $\mathrm{pH} 8.6$ ) solution for $15 \mathrm{~min}$ in order to remove undesired divalent metal ions and rinsed in MilliQ water. Soaking the NTA samples in a solution of $10 \mathrm{mM} \mathrm{NiCl}_{2}$ in $20 \mathrm{mM}$ Tris (pH 7.4) for 5-10 min allowed for the coordination of NTA $\mathrm{COOH}$ groups with $\mathrm{Ni}^{2+}$ ions. The samples were 
then immersed in the salt buffer solution containing a $170 \mu \mathrm{M}$ concentration of $\mathrm{His}_{6}$. A subsets of samples were exposed to

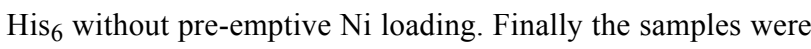
rinsed with the buffer solution, characterized by SE in situ, rinsed in MilliQ water, fluxed with $\mathrm{N}_{2}$ and characterized by SE ex situ. Regeneration of the NTA/Au surface was accomplished by rinsing the sample in $0.4 \mathrm{M}$ imidazole in $20 \mathrm{mM}$ Tris solution and then in $0.5 \mathrm{M}$ EDTA ( $\mathrm{pH} 8.6)$ solution.

\section{Spectroscopic ellipsometry}

SE measurements were performed on a rotating compensator instrument (M-2000, J.A.Woollam Co.Inc.). Ex situ data were collected at $65-70^{\circ}$ angle of incidence. The presented spectra were obtained after the averaging over five samples and over four different zones of each sample. In situ measurements were conducted in a custom-made Teflon cell with $65^{\circ}$ angle of incidence. The in situ data are presented in the transparency region of the solvent. Principles and applications of SE are detailed in books $[42,43]$ and reviews [63,64]. Briefly, standard ellipsometry consists in the investigation of the coefficient $\rho=\tilde{r}_{\mathrm{p}} / \tilde{r}_{\mathrm{s}}=\tan \Psi \mathrm{e}^{i \Delta}$ where $\tilde{r}_{\mathrm{p}}$ and $\tilde{r}_{\mathrm{s}}$ are the complex Fresnel reflection coefficients related respectively to $\mathrm{p}$ - and s-polarization. The ellipsometric analysis is performed through comparison between experimental and simulated data. Simulations are based on models that take into account optical and morphological properties of the layered sample.

\section{AFM nanografting and imaging}

AFM experiments were carried out with a XE100 (Park Instruments) instrument in custom liquid cells. For nanografting, the freshly prepared NTA SAM samples were glued in a custom liquid cell filled with a solution of $30 \mu \mathrm{M}$ HS-C11-OEG6 (T-OEG6) in ethanol. During the nanografting process, we operated in contact mode with commercial AFM tips (NSC19 Mikromasch, $k=0.6 \mathrm{~N} / \mathrm{m})$, applying a high load $(100 \mathrm{nN})$ in a confined (from $0.5 \times 0.5$ to $2 \times 2 \mu \mathrm{m}^{2}$ ) scanning area. The applied load is sufficient to displace the NTA molecules that are substituted by the T-OEG6 molecules present in the ethanol solution $[65,66]$. The nanografted areas have been then imaged in soft contact mode in ethanol or in saline buffer (Tris- $\mathrm{HCl}$ $20 \mathrm{mM}, \mathrm{NaCl} 150 \mathrm{mM}, \mathrm{pH}$ 7.4) with commercial cantilevers (CSC38 Mikromasch, $k=0.03 \mathrm{~N} / \mathrm{m}$ ) at the minimum force detectable $(0.1 \mathrm{nN})$. Images and data were analyzed with XEI (Park Instruments) and Igor Pro (Wavemetrics Inc.) software.

\section{Acknowledgements}

This work was supported by the Italian Ministry of Education (grant RBAP11ETKA-005). The authors thank Matteo Castronovo for useful hints in the initial stage of the work, B. Sanavio for clarifications on the preparation protocols, and G. Bracco for discussions.

\section{References}

1. Prime, K.; Whitesides, G. Science 1991, 252, 1164-1167. doi:10.1126/science.252.5009.1164

2. Hucknall, A.; Rangarajan, S.; Chilkoti, A. Adv. Mater. 2009, 21, 2441-2446. doi:10.1002/adma.200900383

3. Hochuli, E.; Döbeli, H.; Schacher, A. J. Chromatogr. A 1987, 411, 177-184. doi:10.1016/S0021-9673(00)93969-4

4. Gaberc-Porekar, V.; Menart, V. Chem. Eng. Technol. 2005, 28, 1306-1314. doi:10.1002/ceat.200500167

5. Jain, P.; Sun, L.; Dai, J.; Baker, G. L.; Bruening, M. L. Biomacromolecules 2007, 8, 3102-3107. doi:10.1021/bm700515m

6. Kim, S. H.; Jeyakumar, M.; Katzenellenbogen, J. A. J. Am. Chem. Soc. 2007, 129, 13254-13264. doi:10.1021/ja074443f

7. Zhen, G.; Falconnet, D.; Kuennemann, E.; Vörös, J.; Spencer, N. D.; Textor, M.; Zürcher, S. Adv. Funct. Mater. 2006, 16, 243-251. doi:10.1002/adfm.200500232

8. Shen, W.; Zhong, H.; Neff, D.; Norton, M. L. J. Am. Chem. Soc. 2009, 131, 6660-6661. doi:10.1021/ja901407j

9. Liu, Y. C.; Rieben, N.; Iversen, L.; Sørensen, B.; Park, J.; Nygrård, J.; Martinez, K. L. Nanotechnology 2010, 21, 245105. doi:10.1088/0957-4484/21/24/245105

10. Gautrot, J. E.; Huck, W. T. S.; Welch, M.; Ramstedt, M. ACS Appl. Mater. Interfaces 2010, 2, 193-202. doi:10.1021/am9006484

11. Maalouli, N.; Gouget-Laemmel, A. C.; Pinchemel, B.; Bouazaoui, M.; Chazalviel, J.-N.; Ozanam, F.; Yang, Y.; Burkhard, P.; Boukherroub, R.; Szunerits, S. Langmuir 2011, 27, 5498-5505. doi:10.1021/la2005437

12. Valenti, L. E.; Martins, V. L.; Herrera, E.; Torresi, R. M.; Giacomelli, C. E. J. Mater. Chem. B 2013, 1, 4921-4931. doi:10.1039/C3TB20769B

13. Wegner, G. J.; Lee, H. J.; Marriott, G.; Corn, R. M. Anal. Chem. 2003, 75, 4740-4746. doi:10.1021/ac0344438

14. Ataka, K.; Giess, F.; Knoll, W.; Naumann, R.; Haber-Pohlmeier, S.; Richter, B.; Heberle, J. J. Am. Chem. Soc. 2004, 126, 16199-16206. doi:10.1021/ja045951h

15. Valenti, L. E.; Pauli, C. P. D.; Giacomelli, C. E. J. Inorg. Biochem. 2006, 100, 192-200. doi:10.1016/j.jinorgbio.2005.11.003

16. Cheng, F.; Gamble, L. J.; Castner, D. G. Anal. Chem. 2008, 80, 2564-2573. doi:10.1021/ac702380w

17. Ley, C.; Holtmann, D.; Mangold, K.-M.; Schrader, J. Colloids Surf., B 2011, 88, 539-551. doi:10.1016/j.colsurfb.2011.07.044

18. Schartner, J.; Güldenhaupt, J.; Mei, B.; Rögner, M.; Muhler, M.; Gerwert, K.; Kötting, C. J. Am. Chem. Soc. 2013, 135, 4079-4087. doi:10.1021/ja400253p

19. Nakamura, I.; Makino, A.; Ohmae, M.; Kimura, S. Macromol. Biosci. 2010, 10, 1265-1272. doi:10.1002/mabi.201000189

20. Le, T. T.; Wilde, C. P.; Grossman, N.; Cass, A. E. G. Phys. Chem. Chem. Phys. 2011, 13, 5271-5278. doi:10.1039/C0CP02531C

21. Sosna, M.; Boer, H.; Bartlett, P. N. ChemPhysChem 2013, 14 2225-2231. doi:10.1002/cphc.201300340

22. Tinazli, A.; Tang, J.; Valiokas, R.; Picuric, S.; Lata, S.; Piehler, J.; Liedberg, B.; Tampé, R. Chem. - Eur. J. 2005, 11, 5249-5259. doi:10.1002/chem.200500154

23. Valiokas, R.; Klenkar, G.; Tinazli, A.; Reichel, A.; Tampé, R.; Piehler, J.; Liedberg, B. Langmuir 2008, 24, 4959-4967. doi:10.1021/la703709a

24. Khan, F.; He, M.; Taussig, M. J. Anal. Chem. 2006, 78, 3072-3079. doi:10.1021/ac060184| 
25. Hainfeld, J. F.; Liu, W.; Halsey, C. M. R.; Freimuth, P.; Powell, R. D. J. Struct. Biol. 1999, 127, 185-198. doi:10.1006/jsbi.1999.4149

26. Li, Y.-C.; Lin, Y.-S.; Tsai, P.-J.; Chen, C.-T.; Chen, W.-Y.; Chen, Y.-C. Anal. Chem. 2007, 79, 7519-7525. doi:10.1021/ac0711440

27. Xie, H.-Y.; Zhen, R.; Wang, B.; Feng, Y.-J.; Chen, P.; Hao, J. J. Phys. Chem. C 2010, 114, 4825-4830. doi:10.1021/jp910753f

28. Schmitt, L.; Ludwig, M.; Gaub, H. E.; Tampé, R. Biophys. J. 2000, 78, 3275-3285. doi:10.1016/S0006-3495(00)76863-9

29. Wang, C.-H. K.; Jiang, S.; Pun, S. H. Langmuir 2010, 26 , 15445-15452. doi:10.1021/la1025203

30. Uchinomiya, S.; Nonaka, H.; Wakayama, S.; Ojida, A.; Hamachi, I. Chem. Commun. 2013, 49, 5022-5024. doi:10.1039/C3CC41979G

31. Lai, Y.-T.; Chang, Y.-Y.; Hu, L.; Yang, Y.; Chao, A.; Du, Z.-Y.; Tanner, J. A.; Chye, M.-L.; Qian, C.; Ng, K.-M.; Li, H.; Sun, H. Proc. Natl. Acad. Sci. U. S. A. 2015, 112, 2948-2953. doi:10.1073/pnas.1419598112

32. Sigal, G. B.; Bamdad, C.; Barberis, A.; Strominger, J.; Whitesides, G. M. Anal. Chem. 1996, 68, 490-497. doi:10.1021/ac9504023

33. You, C.; Piehler, J. Anal. Bioanal. Chem. 2014, 406, 3345-3357. doi:10.1007/s00216-014-7803-y

34. Poksinski, M.; Arwin, H. Thin Solid Films 2004, 455-456, 716-721. doi:10.1016/j.tsf.2004.01.037

35. Jung, L. S.; Campbell, C. T.; Chinowsky, T. M.; Mar, M. N.; Yee, S. S. Langmuir 1998, 14, 5636-5648. doi:10.1021/la971228b

36. Vanderah, D. J.; Vierling, R. J.; Walker, M. L. Langmuir 2009, 25, 5026-5030. doi:10.1021/la803896a

37. Klenkar, G.; Valiokas, R.; Lundström, I.; Tinazli, A.; Tampé, R.; Piehler, J.; Liedberg, B. Anal. Chem. 2006, 78, 3643-3650. doi:10.1021/ac060024+

38. Nabok, A.; Tsargorodskaya, A. Thin Solid Films 2008, 516, 8993-9001. doi:10.1016/j.tsf.2007.11.077

39. Rodenhausen, K. B.; Kasputis, T.; Pannier, A. K.; Gerasimov, J. Y.; Lai, R. Y.; Solinsky, M.; Tiwald, T. E.; Wang, H.; Sarkar, A.; Hofmann, T.; Ianno, N.; Schubert, M. Rev. Sci. Instrum. 2011, 82, 103111. doi: $10.1063 / 1.3653880$

40. Rauch, S.; Eichhorn, K.-J.; Kuckling, D.; Stamm, M.; Uhlmann, P. Adv. Funct. Mater. 2013, 23, 5675-5681. doi:10.1002/adfm.201300849

41. Azzam, R. M. A.; Bashara, N. M. Ellipsometry and Polarized Light, 3rd ed.; North-Holland: New York, 1977.

42. Fujiwara, H. Spectroscopic Ellipsometry: Principles and Applications, 1st ed.; Wiley: Chichester, United Kingdom, 2007.

43. Tompkins, H.; Irene, E., Eds. Handbook of ellipsometry, 1st ed.; Andrew: Norwich, 2005.

44. Shi, J.; Hong, B.; Parikh, A.; Collins, R.; Allara, D. Chem. Phys. Lett. 1995, 246, 90-94. doi:10.1016/0009-2614(95)01085-N

45. Bordi, F.; Prato, M.; Cavalleri, O.; Cametti, C.; Canepa, M.; Gliozzi, A J. Phys. Chem. B 2004, 108, 20263-20272. doi:10.1021/jp047141y

46. Prato, M.; Moroni, R.; Bisio, F.; Rolandi, R.; Mattera, L.; Cavalleri, O.; Canepa, M. J. Phys. Chem. C 2008, 112, 3899-3906. doi:10.1021/jp711194s

47. Prato, M.; Alloisio, M.; Jadhav, S. A.; Chincarini, A.; Svaldo-Lanero, T.; Bisio, F.; Cavalleri, O.; Canepa, M. J. Phys. Chem. C 2009, 113, 20683-20688. doi:10.1021/jp906298m

48. Toccafondi, C.; Prato, M.; Maidecchi, G.; Penco, A.; Bisio, F.; Cavalleri, O.; Canepa, M. J. Colloid Interface Sci. 2011, 364, 125-132. doi:10.1016/j.jcis.2011.07.097

49. Solano, I.; Parisse, P.; Gramazio, F.; Cavalleri, O.; Bracco, G.; Castronovo, M.; Casalis, L.; Canepa, M. Phys. Chem. Chem. Phys. 2015, 17, 28774-28781. doi:10.1039/C5CP04028K
50. Toccafondi, C.; Cavalleri, O.; Bisio, F.; Canepa, M. Thin Solid Films 2013, 543, 78-82. doi:10.1016/j.tsf.2013.02.117

51. Kang, E.; Park, J.-W.; McClellan, S. J.; Kim, J.-M.; Holland, D. P.; Lee, G. U.; Franses, E. I.; Park, K.; Thompson, D. H. Langmuir 2007, 23, 6281-6288. doi:10.1021/la063719e

52. Canepa, M.; Maidecchi, G.; Toccafondi, C.; Cavalleri, O.; Prato, M.; Chaudhari, V.; Esaulov, V. A. Phys. Chem. Chem. Phys. 2013, 15, 11559-11565. doi:10.1039/C3CP51304A

53. Hamoudi, H.; Prato, M.; Dablemont, C.; Cavalleri, O.; Canepa, M.; Esaulov, V. A. Langmuir 2010, 26, 7242-7247. doi:10.1021/la904317b

54. Hamoudi, H.; Uosaki, K.; Ariga, K.; Esaulov, V. A. RSC Adv. 2014, 4, 39657-39666. doi:10.1039/C4RA05476H

55. Mårtensson, J.; Arwin, H. Langmuir 1995, 11, 963-968. doi:10.1021/la00003a045

56. Hamoudi, H.; Guo, Z.; Prato, M.; Dablemont, C.; Zheng, W. Q.; Bourguignon, B.; Canepa, M.; Esaulov, V. A.

Phys. Chem. Chem. Phys. 2008, 10, 6836-6841. doi:10.1039/b809760g

57. Block, H.; Maertens, B.; Spriestersbach, A.; Brinker, N.; Kubicek, J.; Fabis, R.; Labahn, J.; Schaefer, F. Immobilized-Metal Affinity Chromatography (IMAC): A Review. In Guide to Protein Purification, 2nd ed.; Burgess, R. R.; Deutscher, M. P., Eds.; Methods in Enzymology, Vol. 463; Academic Press, 2009; pp 439-473.

58. Prato, M.; Gussoni, A.; Panizza, M.; Cavalleri, O.; Mattera, L.; Canepa, M. Phys. Status Solidi C 2008, 5, 1304-1307. doi:10.1002/pssc.200777882

59. Toccafondi, C.; Occhi, L.; Cavalleri, O.; Penco, A.; Castagna, R.; Bianco, A.; Bertarelli, C.; Comoretto, D.; Canepa, M. J. Mater. Chem. C 2014, 2, 4692-4698. doi:10.1039/C4TC00371C

60. Thompson, L. B.; Mack, N. H.; Nuzzo, R. G. Phys. Chem. Chem. Phys. 2010, 12, 4301-4308. doi:10.1039/B920713A

61. Vaish, A.; Silin, V.; Walker, M. L.; Steffens, K. L.; Krueger, S.; Yeliseev, A. A.; Gawrisch, K.; Vanderah, D. J. Chem. Commun. 2013, 49, 2685-2687. doi:10.1039/C3CC00077J

62. Gupta, P.; Loos, K.; Korniakov, A.; Spagnoli, C.; Cowman, M.; Ulman, A. Angew. Chem., Int. Ed. 2004, 43, 520-523. doi:10.1002/anie.200352249

63. Losurdo, M.; Bergmair, M.; Bruno, G.; Cattelan, D.; Cobet, C.; de Martino, A.; Fleischer, K.; Dohcevic-Mitrovic, Z.; Esser, N.; Galliet, M.; Gajic, R.; Hemzal, D.; Hingerl, K.; Humlicek, J.; Ossikovski, R.; Popovic, Z. V.; Saxl, O. J. Nanopart. Res. 2009, 11, 1521-1554. doi:10.1007/s11051-009-9662-6

64. Oates, T. W. H.; Wormeester, H.; Arwin, H. Prog. Surf. Sci. 2011, 86, 328-376. doi:10.1016/j.progsurf.2011.08.004

65. Sanavio, B.; Scaini, D.; Grunwald, C.; Legname, G.; Scoles, G.; Casalis, L. ACS Nano 2010, 4, 6607-6616. doi:10.1021/nn101872w

66. Scaini, D.; Castronovo, M.; Casalis, L.; Scoles, G. ACS Nano 2008, 2 , 507-515. doi:10.1021/nn700342p 


\section{License and Terms}

This is an Open Access article under the terms of the Creative Commons Attribution License

(http://creativecommons.org/licenses/by/2.0), which permits unrestricted use, distribution, and reproduction in any medium, provided the original work is properly cited.

The license is subject to the Beilstein Journal of Nanotechnology terms and conditions:

(http://www.beilstein-journals.org/bjnano)

The definitive version of this article is the electronic one which can be found at:

doi:10.3762/bjnano.7.48 